ISSN 0103-9954

\title{
RELAÇ̃̃O DA RAZÃO DO QUOCIENTE DIÂMETRO E ÁREA BASAL COM O AUTODESBASTE EM POVOAMENTOS EQUIÂNEOS DE Pinus taeda L.
}

\author{
DIAMETER AND BASAL AREA RATIO RELATIONSHIP WITH SELF-THINNING IN \\ Pinus taeda L. EVEN-AGED STANDS
}

\author{
Paulo Sérgio Pigatto Schneider ${ }^{1}$ César Augusto Guimarães Finger ${ }^{2}$ \\ Paulo Renato Schneider ${ }^{3}$
}

\begin{abstract}
RESUMO
Este trabalho teve por objetivo estudar a relação do diâmetro médio e da área basal no momento do autodesbaste, o crescimento diamétrico e a ocorrência do autodesbaste, o momento de autodesbaste com base na proporção de diâmetro-área basal, a taxa de autodesbaste comparando-a com a lei da potência -3/2 em povoamentos de Pinus taeda L., em vários espaçamentos, manejado em densidade completa. O modelo de Tang foi empregado relacionando o número de árvores por hectare com o diâmetro médio, a partir de dados de parcelas permanentes, medidas anualmente até os 18 anos em experimento de espaçamento. $\mathrm{O}$ modelo de autodesbaste mostrou ótima precisão e elevada eficiência, porém, apresentou coeficiente angular de $-1,65$ com inclinação diferente da preconizada na lei de autodesbaste de $-3 / 2$. No momento da ocorrência do autodesbaste, as árvores atingiram diâmetros 14 e $17 \mathrm{~cm}$, nos espaçamento de 2 x 2 e 3 x 2 m, com áreas basais de 38,8 e 47,5 m²/ha, respectivamente. Em todos os espaçamentos a área basal máxima foi de $78 \mathrm{~m}^{2} /$ ha, aos 18 anos de idade, apresentando ainda tendência ascendente. Nesses espaçamentos, o quociente do diâmetro pela área basal no momento de ocorrência do autodesbaste foram 0,36 e 0,37 , respectivamente. Palavras-chave: diâmetro; área basal; autodesbaste; densidade.
\end{abstract}

\begin{abstract}
This work aimed to study the relationship of the ratio of mean diameter and basal area at the self-thinning moment; the diameter tree growth; the occurrence and moment of self-thinning using the diameter-area proportion and the rate of self-thinning, comparing it to the law of the $-3 / 2$ power in stands of Pinus taeda L.. The data was collected from permanent plots experiment installed into a Pinus taeda L. stand and measured annually until 18 years-old with different spacing managed with complete density. The Tang model was used in order to relate the density of trees with the mean diameter. The self-thinning model showed precision and high efficiency. However, it showed a different slope coefficient of -1.65 that stressed from the selfthinning law of $-3 / 2$. At the self-thinning moment, the trees reached a diameter of 14 and $17 \mathrm{~cm}$ at the space of $2 \times 2$ and $3 \times 3$ with basal area of 38.8 and $47.5 \mathrm{~m}^{2} /$ ha, respectively. In all spacing the maximum basal area was $78 \mathrm{~m}^{2} /$ ha at 18 years old showing upward trend. In that spacing the diameter and basal area ratio at the moment of occurrence of self-thinning was 0.36 and 0.37 , respectively.
\end{abstract}

Keywords: diameter; basal area; self-thinning; density.

1 Engenheiro Florestal, Msc., Doutorando pelo Programa de Pós-Graduação em Engenharia Florestal, Centro de Ciências Rurais, Universidade Federal de Santa Maria, Av. Roraima, 1000, CEP 97105-900, Santa Maria (RS), Brasil.psps@mail.ufsm.br

2 Engenheiro Florestal, Dr., Professor Associado do Departamento de Ciências Florestais, Centro de Ciências Rurais, Universidade Federal de Santa Maria, Av. Roraima, 1000, CEP 97105-900, Santa Maria (RS), Brasil. finger@smail.ufsm.br

3 Engenheiro Florestal, Dr., Professor Titular do Departamento de Ciências Florestais, Centro de Ciências Rurais, Universidade Federal de Santa Maria, Av. Roraima, 1000, CEP 97105-900, Santa Maria (RS), Brasil. paulors@smail.ufsm.br

Recebido para publicação em 2/05/2011 e aceito em 19/09/2012

Ci. Fl., v. 24, n. 2, abr.-jun., 2014 


\section{INTRODUÇÃO}

A competição intraespecífica é um importante fator que afeta o tamanho médio das plantas em uma população e, por isto, tem sido estudada buscando descrever a relação entre o tamanho das plantas e a densidade da população, bem como a sua evolução no tempo.

O conceito de densidade de manejo de um povoamento é um processo de controle do crescimento do estoque inicial ou do estoque após desbastes para atender a objetivos específicos de manejo e de produção de matéria prima. A determinação do nível apropriado do estoque de crescimento para um nível de povoamento é um processo complexo com envolvimento biológico, tecnológico e de fatores econômicos específicos para uma dada situação. $\mathrm{O}$ processo requer a seleção de limites inferiores e superiores de estoque de crescimento em uma determinada ocupação do sítio, visando a um adequado nível de produção, com um número de árvores desejado.

De acordo com Reineke (1933), em povoamento com densidade completa, a relação entre o número de árvores por hectare $(\mathrm{N})$ e o diâmetro quadrático médio (dg) é linear numa escala dupla logarítmica e que os parâmetros da função independem da idade e da qualidade do sítio. Postulou o autor que, a relação entre o número de árvores por hectare e o diâmetro médio quadrático em povoamento sem tratamento é uma linha reta, com um valor do coeficiente angular constante de $-1,605$.

Del Rio et al. (2001) analisaram a relação do diâmetro e densidade populacional, com referência ao estabelecimento do autodesbaste em povoamento equiâneo não desbastado de Pinus silvestris. Os resultados, empregando as funções de Reineke (1933), Zeide (1995) e Tang et al. (1994), indicaram que o autodesbaste seguiu uma linha côncava com incremento do coeficiente angular quando do aumento da densidade do povoamento, assumindo a assíntota na linha de densidade máxima, com coeficiente angular igual a $-1,75$.

A lei da potencia $-3 / 2$ de autodesbaste desenvolvida, entre as décadas 1960 e 1970, por cientistas japoneses para colheitas agrícolas (YODA et al., 1963) descreve que para qualquer densidade há um máximo de biomassa comum que uma planta individualmente pode atingir. Um aumento adicional de biomassa da planta só pode ser alcançado a uma densidade populacional mais baixa sendo, para isso, necessário que algumas plantas morram. Essa relação, inicialmente descrita pelo logaritmo do volume médio das árvores e o número de árvores por hectare, é linear com coeficiente angular -1,5 e define uma linha de proporção máxima do número de árvores pelo tamanho, com plantas de mesma taxa de crescimento, para qualquer local. Nesta situação o autodesbaste deve ocorrer quando as árvores tiverem uma completa ocupação do sítio.

Tang et al. (1994) estenderam a teoria do autodesbaste para povoamentos em densidade completa e subestocados constatando que o autodesbaste começa antes de o povoamento atingir a máxima densidade, e que a taxa de autodesbaste incrementa com a densidade, atingindo maior valor em povoamento de densidade máxima. No modelo definido pelos autores, a lei do autodesbaste pode ser considerada um caso especial, pois permite obter ainda para qualquer diâmetro, a densidade e a produção.

A lei do autodesbaste tem sido objeto de uso prático e de discussões acerca da sua eficiência por vários pesquisadores, como: Westoby (1981), West e Bourough (1983), Zeide (1985, 1987 e 1991), Smith e Hann (1986), Sterba e Monserud (1993), Yoda et al. (1963), Tang et al. (1994), Osawa e Allen (1993), Tang et al. (1995), Del Rio et al. (2001), entre outros.

Curtis (1982) utilizou o princípio e a equação de Reineke (1933) para descrever a relação entre a área basal $(G)$ e o diâmetro quadrático médio (dg) definindo para povoamentos de Pseudotsuga menziensii um valor para o coeficiente angular entre 0,45 e 0,5 , mas optando pelo valor 0,5 para determinar a densidade relativa e absoluta do povoamento. Recentemente, Reid (2006), com dados de plantações da Austrália e Nova Zelândia, demonstrou o uso do quociente do diâmetro médio das árvores e a área basal do povoamento (d:G) para o manejo de florestas não industriais em pequenas propriedades rurais concluindo que o quociente é simples e prático para descrever o estoque de plantações de Eucalyptus, e que a manutenção desse quociente constante levou ao aumento da competição com o aumento da idade.

Assim, esse trabalho foi desenvolvido visando definir o valor do diâmetro médio e da área basal no momento do autodesbaste; o crescimento diamétrico e a ocorrência do autodesbaste; o momento de autodesbaste com base na proporção de diâmetro-área basal, a taxa de autodesbaste, comparando-a com a lei da potência $-3 / 2$. 


\section{MATERIAIS E MÉTODOS}

\section{Localização e caracterização da área de estudo}

Os dados foram levantados em florestas pertencentes à Klabin S.A., no município de Otacílio Costa, região fisiográfica do Planalto Central Catarinense (SANTA CATARINA, 1986).

O clima da região é do tipo "Cfb", mesotérmico, subtropical úmido, com verões frescos, não apresentando estação seca definida, segundo a classificação climática de Köppen, A precipitação média anual é de $1.808 \mathrm{~mm}$, distribuída em 124 dias, temperatura média anual de $17,8^{\circ} \mathrm{C}$ (MOTTA et al., 1971).

O solo da área do experimento é classificado como latossolo bruno, alumínico TB, horizonte A húmico, textura argilosa e relevo suavemente ondulado.

Os dados foram medidos em experimento concebido no Modelo Nelder Modificado, instalado em 1988 e remedido periodicamente até a idade de 18 anos. Em cada unidade amostral foram medidas as circunferências a altura do peito e a altura das árvores. $\mathrm{O}$ delineamento estatístico foi em blocos ao acaso, com três repetições e nove tratamentos, sendo estes definidos pelo espaçamento inicial de plantio. Destes tratamentos foram utilizados os de: $1,5 \times 1,0\left(1,5 \mathrm{~m}^{2}\right), 2,5 \times 1,0\left(2,5 \mathrm{~m}^{2}\right), 1,5 \times 2,0\left(3 \mathrm{~m}^{2}\right)$, $2,0 \times 2,0\left(4 \mathrm{~m}^{2}\right), 1,5 \times 3,0\left(4,5 \mathrm{~m}^{2}\right), 2,5 \times 2,0\left(5 \mathrm{~m}^{2}\right)$, $2,0 \times 3,0\left(6 \mathrm{~m}^{2}\right), 2,5 \times 3,0\left(7,5 \mathrm{~m}^{2}\right)$.

Os dados referentes ao número de árvores por hectare e diâmetro médio tiveram origem em parcelas permanentes localizadas em povoamentos implantados com vários espaçamentos, medidas periodicamente tendo originado, aproximadamente, 50 observações em cada espaçamento.

\section{Metodologia empregada}

O estudo da densidade e diâmetro e do autodesbaste foi realizado a partir do número de árvores por hectare e o correspondente diâmetro médio, determinado nas unidades amostrais e ajustados com o procedimento NLIN, pelo método Gauss-Newton, no pacote SAS (1999). O modelo do autodesbaste empregado foi o proposto por Tang et al. (1994), sendo expresso por:

$$
\ln N(t)=\ln S_{f}-\frac{1}{\gamma} \ln \left[\left(D(t) / d_{o}\right)^{\beta \gamma}+\delta\right]
$$

Sendo $t$ a idade do povoamento (ano), a taxa máxima de autodesbaste em densidade completa, $\mathrm{N}(\mathrm{t})$ a densidade no tempo $\mathrm{t}$ (número de árvores por hectare), $\mathrm{D}(\mathrm{t})$ o diâmetro médio das árvores no tempo $\mathrm{t}(\mathrm{cm}), \mathrm{S}_{\mathrm{f}}$ o índice da máxima densidade, $\mathrm{o}$ índice de autodesbaste, e $\mathrm{d}_{\mathrm{o}} \mathrm{o}$ diâmetro básico padrão $(25 \mathrm{~cm})$. A constante determinada pela densidade inicial do povoamento $\left(\mathrm{N}_{1}\right)$ e o diâmetro médio inicial $\left(\mathrm{d}_{1}\right)$, pela seguinte fórmula:

$$
\delta=\left(S_{f} / N_{1}\right)^{\gamma}-\left(d_{1} / d_{o}\right)^{\beta \gamma}
$$

O modelo de Tang foi calculado de forma independente para cada espaçamento inicial e, posteriormente, recalculado para os dados dos dois espaçamentos de maior densidade de plantio, respectivamente, $1,5 \times 1,0 \mathrm{~m}$ e 2,5 x 1,0 m.

Com os novos coeficientes obtidos para os dois espaçamentos, representando a máxima densidade, foram geradas as curvas de densidade ajustadas para cada densidade de plantio permitindo identificar o estabelecimento do autodesbaste e a dimensão do diâmetro naquela ocasião.

Para verificar a precisão estatística dos modelos de densidade de árvores e diâmetro foi determinado o coeficiente de determinação ajustado, erro padrão da estimativa. Para validação utilizaramse as estatísticas da tendência (Bias) e eficiência (E) dos modelos, recomendados por Palahi et al. (2002) e Cellini et al. (2002).

\section{RESULTADOS E DISCUSSÃO}

Os parâmetros estatísticos do modelo de autodesbaste de Tang gerados com o diâmetro e a densidade de árvores por hectare nos espaçamentos de plantio: $1,5 \times 1,0 \mathrm{~m}\left(1,5 \mathrm{~m}^{2}\right), 2,5 \times 1,0\left(2,5 \mathrm{~m}^{2}\right)$, $1,5 \times 2,0\left(3 \mathrm{~m}^{2}\right), 2,0 \times 2,0\left(4 \mathrm{~m}^{2}\right), 1,5 \times 3,0\left(4,5 \mathrm{~m}^{2}\right)$, $2,5 \times 2,0\left(5 \mathrm{~m}^{2}\right), 2,0 \times 3,0\left(6 \mathrm{~m}^{2}\right), 2,5 \times 3,0\left(7,5 \mathrm{~m}^{2}\right)$, mostraram excelente ajuste e baixo erro padrão da estimativa, sem tendência, expressa no valor de bias inferior a 0,009 e alta eficiência, com valor superior a 0,999 , em todos os espaçamentos (Tabela 1).

A análise dos valores do parâmetro, que remete para a interpretação e comprovação da lei do autodesbaste, evidenciaram sua grande variação com o espaçamento de plantio, sem ter mantido uma tendência lógica de valores. Da mesma forma, os parâmetros calculados diferiram do preconizado por Tang et al. (1994) como igual a $-3 / 2$, para todas as situações de manejo e tratamento silvicultural. No espaçamento mais denso (1,5 x 1,0 m), o valor deste 
TABELA 1: Estatísticas do modelo de densidade e diâmetro de Tang, ajustado por espaçamento.

TABLE 1: Statistical results of the size-density model per the diameter of Tang, adjusted by spacing.

\begin{tabular}{|c|c|c|c|c|c|c|c|}
\hline \multirow{2}{*}{ Espaçamento (m) } & \multicolumn{3}{|c|}{ Parâmetros } & \multirow{2}{*}{$\mathrm{R}^{2}$} & \multirow{2}{*}{$S_{y x}$} & \multirow{2}{*}{ Bias } & \multirow{2}{*}{$\mathrm{E}$} \\
\hline & $S_{f}$ & $\beta$ & $\gamma$ & & & & \\
\hline $1,5 \times 1,0$ & 1378,3 & 1,9578 & 5,4411 & 0,9998 & 0,0595 & 0,0031 & 0,9997 \\
\hline $2,5 \times 1,0$ & 1354,9 & 1,6886 & 16,5602 & 0,9998 & 0,0549 & 0,0028 & 0,9997 \\
\hline $1,5 \times 2,0$ & 1213,0 & 1,7784 & 14,3297 & 0,9998 & 0,0495 & 0,0017 & 0,9999 \\
\hline $2,0 \times 2,0$ & 1406,8 & 1,3864 & 23,3919 & 0,9999 & 0,0274 & 0,0007 & 0,9999 \\
\hline $1,5 \times 3,0$ & 1595,9 & 1,9100 & 4,5000 & 0,9999 & 0,0558 & 0,0007 & 0,9999 \\
\hline $2,5 \times 2,0$ & 1352,8 & 1,2271 & 59,2456 & 0,9998 & 0.0423 & 0,0016 & 0,9998 \\
\hline $2,0 \times 3,0$ & 1401,0 & 1,2000 & 54,7276 & 0,9999 & 0,0276 & 0,0015 & 0,9998 \\
\hline $2,5 \times 3,0$ & 1159,9 & 1,7000 & 7,5600 & 0,9999 & 0,1014 & 0,0099 & 0,9998 \\
\hline
\end{tabular}

Fonte: Schneider (2008).

coeficiente foi igual 1,9578 .

Os coeficientes calculados nos espaçamentos permitiram obter uma curva decrescente da densidade de árvores por hectare, representando a máxima densidade por hectare e, assim, a capacidade de tolerância fisiológica das árvores para se manterem vivas na população. Nessa condição, passou a ocorrer o autodesbaste, porque, a partir deste ponto, as árvores passaram a não suportar mais a concorrência ocorrendo, normalmente, a morte das árvores mais dominadas da população.

$\mathrm{O}$ ajuste do modelo de densidade e diâmetro de Tang et al. (1994) padronizada para o diâmetro de referência de $25 \mathrm{~cm}$ e calculada com os dados observados nas parcelas permanentes dos espaçamentos $1,5 \times 1,0$ e $2,5 \times 1,0 \mathrm{~m}$, igualmente apresentou um ótimo ajuste, com um coeficiente de determinação de 0,99 , erro padrão da estimativa de 0,0948 e baixo coeficiente de variação, $1,17 \%$, comprovando a sua eficiência neste tipo de ajuste de dados.

$$
\ln N=\ln 1494,5-\frac{1}{16,56} \ln \left[\begin{array}{l}
(d / 25)^{1,6523} 3^{* 16,56}+ \\
\left(1494,5 / N_{1}\right)^{16,56}-\left(d_{1} / d_{0}\right)^{1,6523 * 16,56}
\end{array}\right],
$$

Em que, d é o diâmetro médio das árvores, $\mathrm{d}_{1}$ é o diâmetro médio inicial, $\mathrm{d}_{0}$ é o diâmetro básico padrão $(25 \mathrm{~cm})$ e $\mathrm{N}_{1}$ a densidade de árvores iniciais.

Com o modelo foram geradas curvas decrescentes representando a máxima densidade de árvores por hectare ou, de outra forma, a máxima capacidade de tolerância fisiológica das árvores para se manterem vivas no habitat (Figura 1). Sobre essas linhas pode-se obter a dimensão do diâmetro médio quando iniciou o autodesbaste, e ainda verificar que esse diâmetro variou, consideravelmente, com a densidade de árvores de plantio, sendo sempre maior quanto maior for o espaçamento inicial, como demonstrado na Figura 2.

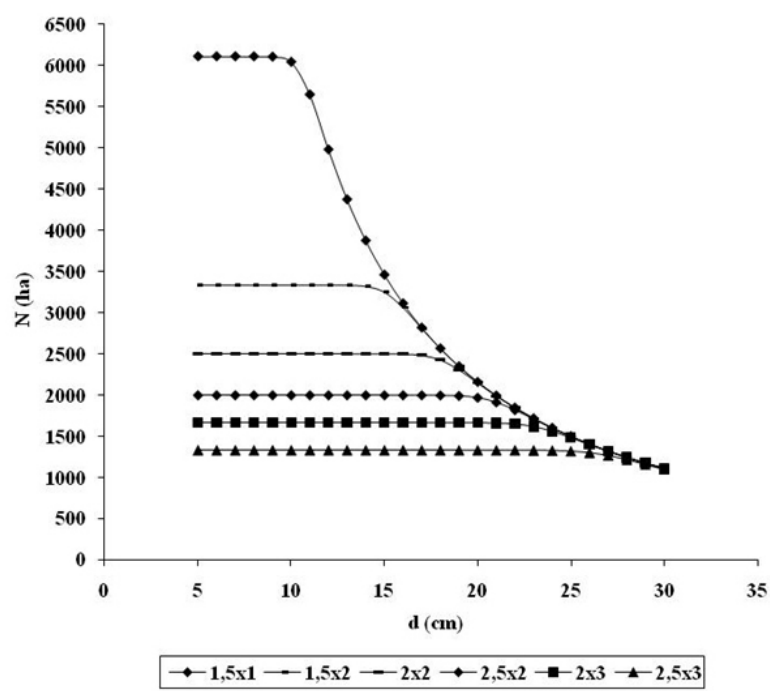

FIGURA 1: Tendências do autodesbaste, definido pela densidade de árvores por hectare em função do diâmetro médio, obtidas com o modelo proposto por Tang et al. (1994).

FIGURE 1: Self-thinning tendencies, defined by the density of trees per hectare in function of the average diameter, obtained from the model proposed by Tang et al. (1994).

No povoamento implantado em espaçamento de $2 \times 2$ ou $3 \times 2$ m o autodesbaste ocorreu no momento em que no diâmetro médio 
atingiu valores de 14 e $19 \mathrm{~cm}$, respectivamente. A partir desse momento estabeleceu-se uma expressiva concorrência e, por consequência, mortalidade de árvores, permitindo inferir que a execução de desbaste deveria ocorrer antes do início da mortalidade natural, evitando-se perdas de produção.

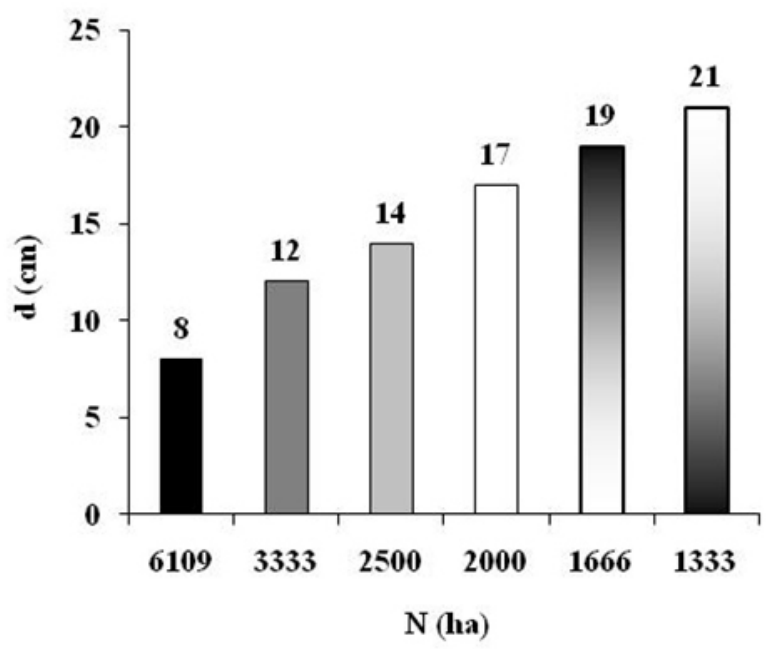

$\square 1,5 \times 1 \quad \square 1,5 \times 2 \quad \square 2 \times 2 \quad \square 2,5 \times 2 \quad \square 2 \times 3 \quad \square 2,5 \times 3$

FIGURA 2: Diâmetro médio aritmético no momento da ocorrência do autodesbaste em relação do número de árvores por hectare do espaçamento inicial.

FIGURE 2: Mean diameter at the moment of the occurrence of self-thinning in relation with the number of trees per hectare in the initial spacing.

Nestas condições, a relação do quociente do diâmetro médio pela área basal por hectare no momento de ocorrência do autodesbaste, representado na Figura 3, mostrou que o valor desse quociente variou significativamente com o espaçamento inicial de implantação do povoamento, onde nos espaçamentos de $2 \times 2$ e $3 \times 2 \mathrm{~m}$, os valores foram iguais a 0,36 e 0,40 .

A área basal por hectare estimada com o diâmetro médio de cada espaçamento e o número de árvores estimadas com o modelo de Tang (Figura 4) mostrou que esta cresceu numa relação direta com o diâmetro médio da população, porém, em níveis estratificados segundo a densidade de plantio numa relação inversa com o espaço vital disponível às árvores. Em todos os espaçamentos utilizados os valores tenderam a aproximar-se da área basal máxima, acima de $78 \mathrm{~m}^{2} / \mathrm{ha}$, porém, mantendo a tendência de incremento ascendente.

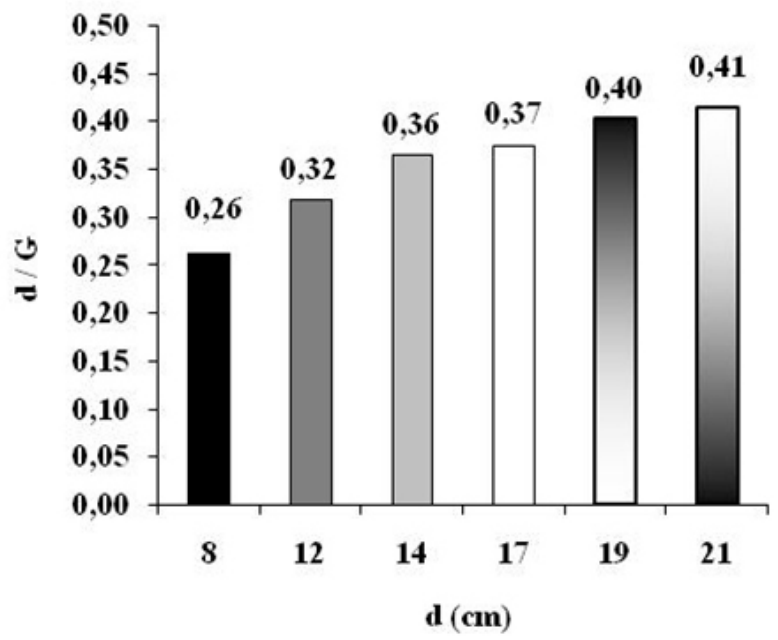

$\square 1,5 \times 1 \quad \square 1,5 \times 2 \quad \square 2 \times 2 \quad \square 2,5 \times 2 \quad \square 2 \times 3 \quad \square 2,5 \times 3$

FIGURA 3: Quociente do diâmetro pela área basal no momento da ocorrência do autodesbaste por espaçamento inicial.

FIGURE 3: Diameter: basal area ratio at the moment of occurrence of self-thinning per initial spacing.

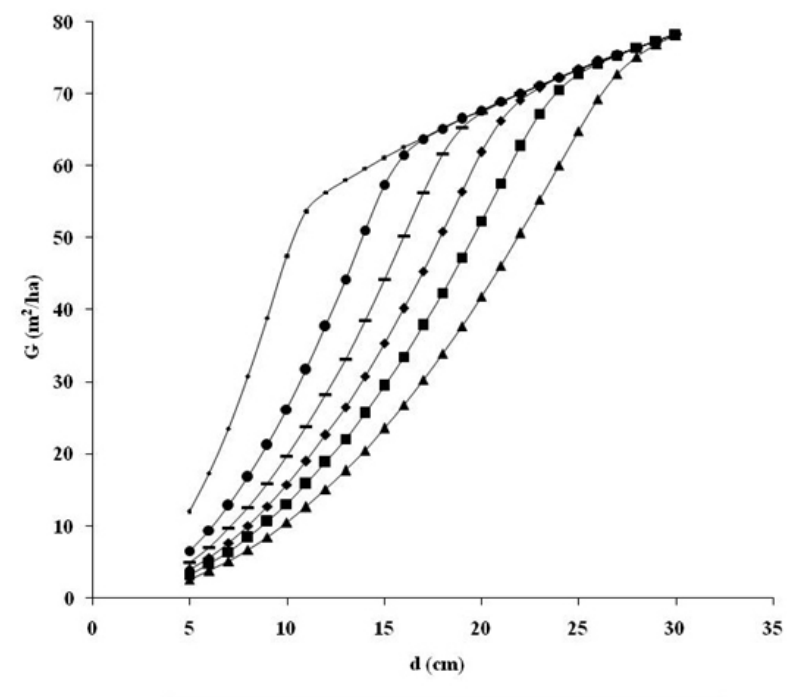

$\rightarrow-1,5 \times 1 \rightarrow-1,5 \times 2-2 \times 2 \rightarrow-2,5 \times 2-2 \times 2 \times 3-2,5 \times 3$

FIGURA 4: Desenvolvimento da área basal em relação ao diâmetro por espaçamento inicial.

FIGURE 4: Development of the basal area in relation with the diameter per initial spacing.

O desenvolvimento do quociente do diâmetro médio pela área basal em todos os 
espaçamentos testados resultou em valores com grande variação, entre 0,3 a 2,0 , observados a partir dos espaçamentos reduzidos aos mais amplos (Figura 5). Esse quociente descreveu uma curva com forma exponencial negativa em relação ao diâmetro médio, estabelecendo diferenças em níveis, tendo ocorrido sua estabilização em torno do valor 0,5 , encontrado, aproximadamente, no momento em que as curvas se aproximaram da curva do espaçamento mais reduzido, ou seja, com espaço vital de $1,5 \mathrm{~m}^{2}$ por ocasião do plantio.

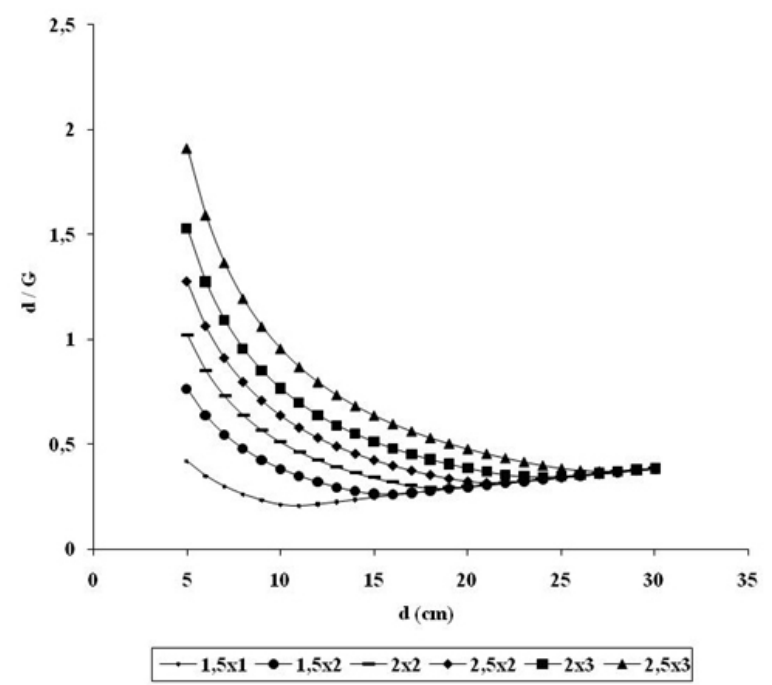

FIGURA 5: Quociente do diâmetro pela área basal em relação ao diâmetro por espaçamento inicial.

FIGURE 5: Diameter: basal area ratio in relation with the diameter per initial spacing.

\section{CONCLUSÕES}

O modelo de autodesbaste de Tang apresenta ótima precisão estatística e elevada eficiência, com coeficiente angular de $-1,65$, distinto do valor $-3 / 2$ prescrito para lei de autodesbaste;

O diâmetro médio do momento da ocorrência do autodesbaste na população varia com a densidade de árvores de plantio alcançando valores de 14 e $17 \mathrm{~cm}$, nos espaçamentos de 2 x 2 ou $3 \times 2$ m, respectivamente;

A relação entre o diâmetro médio pela área basal no momento de ocorrência do autodesbaste varia com o espaçamento inicial com tendência a aproximar-se aos espaçamentos de $2 \times 2$ e $3 \times 2 \mathrm{~m}$, com valores de 0,36 e 0,37 , respectivamente;

Todos os espaçamentos utilizados tendem a aproximar-se da área basal máxima, em torno de
$78 \mathrm{~m}^{2} /$ ha, aos 18 anos de idade, porém, nesta idade, ainda mostra tendência de incremento ascendente até a idade considerada;

$\mathrm{O}$ quociente do diâmetro médio pela área basal numa dimensão temporal em todos os espaçamentos varia entre valores de 0,3 a 2,0, entre os espaçamentos reduzidos aos mais amplos, estabelecendo diferentes níveis, com estabilização em torno do quociente 0,5 , momento em que ocorre o autodesbaste.

\section{AGRADECIMENTOS}

Os autores agradecem a Klabin S.A., pela concessão dos dados dendrométricos, em especial aos engenheiros Antônio Maurício Moreira e Djalma Milles Chaves, responsáveis pela instalação e condução do experimento de espaçamento.

\section{REFERÊNCIAS BIBLIOGRÁFICAS}

CELLINI, J. M. et al. Modelos de perfil de tronco en Nothofagus pumilio (Polp, et Endl,) Krasser y su utilización en el cálculo del volumen total. Invest. Agr, Sist. Recur. For. Ministerio de Agricultura, Instituto Forestal de Investigaciones y Experiencias, Madrid, n. 2, v.11, p. 245-261, 2002.

CURTIS, R. O. A simple index of stand density for Douglas-fir. Forest Science, Bethesda, n. 1, v. 28, p. 92-94, March, 1982.

DEL RIO, M.; MONTERO, G.; BRAVO, F. Analysis of diameter-density relationships and self-thinning in non-thinned even-aged Scots pine stands. Forest Ecology and Management. Amsterdan, v. 142, p. 79-87, March, 2001.

EMBRAPA. Sistema brasileiro de classificação de solos. Brasília: Embrapa, 1999, 412 p.

MOTTA, F. S.; BEIRSDORF, M. J. C.; GARCEZ, R. B. Zoneamento agrícola do Rio Grande do Sul e Santa Catarina: normas agro-climáticas. Pelotas: Ministério da Agricultura. 1971. 80 p.

OSAWA, A.; ALLEN, R. B. Allometric theory explains self-thinning relationships of mountain beech and Red pine. Ecology. n. 74, v. 4, p. 1020-1032, Apr. 1993.

PALAHI, M.; MIIMA, J.; MONTERO, E. Standlevel yield model for scots pine (Pinus sylvestris) in north-east Spain. Invest. Agr. Sist. Recr. For. Ministerio de Agricultura, Instituto Forestal de Investigaciones y Experiencias, Madrid, v. 11, n. 2, p. 409-424, 2002. 
REID, R. Diameter-basal area ratio as a practical stand density measure for pruned plantations. Forest Ecology and Management. Amsterdan, v. 233, p. 375-382, Sept. 2006.

REINEKE, L. H. Perfecting a stand-density index for even-aged forests. Canadian Agricultural Research. Ottawa, n. 46, p. 627-638, 1933.

SANTA CATARINA. Gabinete de Planejamento e Coordenação Geral. Subchefia de Estatística. Geografia e Informática. Atlas de Santa Catarina. Rio de Janeiro: Aerofoto Cruzeiro. 1986. 173 p.

SAS Institute Inc. SAS/STAT user's guide. Version 8 (computer manual). SAS Institute Inc., Cary. N.C. 1999.

SCHNEIDER, P. S. P. Autodesbaste e diagrama de manejo da densidade em povoamento de Pinus taeda L. 2008. 94 f. Dissertação (Mestrado em Engenharia Florestal) - Universidade Federal de Santa, Santa Maria, 2008.

SMITH, N. J.; HANN, D. W. A growth model based on the self-thinning rule. Canadian Journal of Forest Research. New Westminster, v. 16, n. 2, p. 330-334, Apr. 1986.

STERBA, H.; MONSERUD, R. A. The maximum density concept applied to uneven-aged mixedspecies stands. Forest Science. Bethesda, v. 39, n. 3, p. 432-452, Aug. 1993.

TANG, S. et al, A growth and self-thinning model for pure even-aged stands: theory and applications. Forest Ecology and Management. Amsterdan, v. 70, p. 67-73, Dec. 1994.

TANG, S.; MENG, F. R.; MENG, C. H. The impact of initial stand density and site index on maximum stand density index and self-thinning index in a stand self-thinning model. Forest Ecology and Management. Amsterdan, v. 75, p. 61-68, June, 1995.

WEST, P. W.; BOUROUGH, C. J. Tree supervision and the self-thinning, Rule in a monoculture of Pinus radiata D, Don. Annals of Botany. Oxford, v. 52, p. 149-158, 1983.

WESTOBY, M. The place of the self-thinning rule in population dynamics. American Naturalist. v. 118, n. 4, p. 581-587, Oct. 1981.

YODA, K.; KIRA, T.; OGAWA, H.; HOZUMI, K. Self-thinning in over crowed pure stands under cultivated and natural conditions. Jor. Biol. Osaka City Univ. Osaka, v. 14, p. 107-129, 1963.

ZEIDE, B. A relationship between size of trees and their number. Forest Ecology and Management. Amsterdan, v. 72, p. 265-272, Apr. 1995.

ZEIDE, B. Analysis of the $3 / 2$ power law of selfthinning. Forest Science. Bethesda, v. 33, n. 2, p. 17-537, June, 1987.

ZEIDE, B. Self-thinning and stand density. Forest Science. Bethesda, v. 37, n. 2, p. 517-523, June, 1991.

ZEIDE, B. Tolerance and self-tolerance of trees.

Forest Ecology and Management. Amsterdan, v. 13, p. 149-166, Nov. 1985. 